\title{
On fuzzy approximation operators and fuzzy transformation systems
}

\author{
Sutapa Mahato ${ }^{a}$ and S.P. Tiwari ${ }^{b}$ \\ ${ }^{a}$ Department of Applied Mathematics, IIT(ISM), Dhanbad-826004, India, sutapaiitdhanbad@gmail.com \\ ${ }^{b}$ Department of Applied Mathematics, IIT(ISM), Dhanbad-826004, India, sptiwarimaths@gmail.com
}

\begin{abstract}
The objective of this paper is to establish the relationship between fuzzy approximation operators and fuzzy transformation systems. We show that for each upper/lower fuzzy transformation system there exits a fuzzy approximation space induced by a fuzzy reflexive relation and vice-versa.
\end{abstract}

Keywords: Fuzzy approximation space, Relation preserving map, Fuzzy backward natural transformation, Fuzzy transformation systems.

\section{Introduction}

The concept of rough set was originally proposed by Pawlak [11]. This theory has been developed significantly due to its importance for the study of intelligent systems with insufficient and incomplete information. In rough sets introduced by Pawlak, the key role is played by equivalence relations. In literature $[7,16,25]$, several generalizations of rough sets have been made by replacing the equivalence relation by an arbitrary relation. After Dubois and Prade [5] introduced the concept of fuzzy rough set, which is a generalization of rough set. Recently, the combinations of fuzzy sets and rough sets were investigated with different fuzzy logic operations and binary fuzzy relation in $[4,8,10,17,18,21,22,23,24]$, where fuzzy implications play an important role in the extensions of fuzzy rough sets.

Fuzzy transform (F-transform in short), firstly proposed by Perfilieva [13], has now been significantly developed and opened a new page in the theory of semi-linear spaces. The main idea of the $F$-transform is to factorize (or fuzzify) the precise values of dependent variables are averaged to an approximate value.
It is shown in [13] that this transform encompassed both classical transform as well as approximation methods based on fuzzy IF-THEN rules studied in fuzzy modeling. The theory of $F$-transform was further elaborated and extended from real valued to lattice valued functions $[13,14]$ and from fuzzy sets to parametrized fuzzy sets [20]. Recently in [15], it is shown that $F$-transform is a realization of an abstract fuzzy rough set theory, more precisely, $F$-transforms turn out to be approximation operators studied in fuzzy rough set theory.

The concepts of upper and lower fuzzy transformation systems were introduced recently by Močkor [9] and a close connection with $F$-transforms is obtained. Specifically, it is shown that a transformation function satisfies axioms for fuzzy upper (or lower, respectively) transformation systems if and only if it is an upper (or lower, respectively) $F$-transform.

In view of the fact that (i) an $F$-transform can be viewed as a fuzzy approximation operator, and (ii) there is a bijective correspondence between an $F$-transform and a fuzzy transformation system, it is natural to think about the relationship between a fuzzy approximation operator and a fuzzy transformation system. The answer of this problem is theme of this work.

The paper is organized as follows. In Section 2, we recall some basic properties of residuated lattice and fuzzy sets. Fuzzy upper and lower approximation spaces and their properties are discussed in Section 3. In Section 4, we introduce upper and lower fuzzy backward natural transformations and discuss the connection between them. In the next section, we study a relationship between fuzzy transformation systems and fuzzy approximation spaces. At last, we conclude our research in Section 6. 


\section{Preliminaries}

In this section, we recall some concepts related to residuated lattices and fuzzy sets. For details on residuated lattices and fuzzy sets, we refer the works of $[1,2,3,6,12,19,26]$. We begin with the following.

Definition 2.1 $A$ residuated lattice is an algebra $(L, \wedge, \vee, \otimes, \rightarrow, 0,1)$ such that

(i) $(L, \wedge, \vee, 0,1)$ is a bounded lattice with the least element 0 and the greatest element 1 ;

(ii) $(L, \otimes, 1)$ is a commutative monoid; and

(iii) $\forall a, b, c \in L ; a \otimes b \leq c$ iff $a \leq b \rightarrow c$, i.e., $(\rightarrow, \otimes)$ is an adjoint pair on $L$.

$A$ residuated lattice $(L, \wedge, \vee, \otimes, \rightarrow, 0,1)$ is complete if it is complete as a lattice.

Definition 2.2 Let $L=(L, \wedge, \vee, \otimes, \rightarrow, 0,1)$ be $a$ residuated lattice. A negation in $L$ is a unary operation $\neg$ defined by $\neg a=a \rightarrow 0, \forall a \in L . L$ is said to satisfy the law of double negation if $a=\neg(\neg a), \forall a \in L$.

Proposition 2.1 Let $(L, \wedge, \vee, \otimes, \rightarrow, 0,1)$ be a complete residuated lattice. Then for all $a, b, c \in L$,

$$
\begin{aligned}
& \text { 1. } a \leq b \Rightarrow a \otimes c \leq b \otimes c, \\
& \text { 2. If } a \leq b \text {, then } b \rightarrow c \leq a \rightarrow c, \\
& \text { 3. } 1 \otimes a=a \otimes 1=a, \\
& \text { 4. } a \otimes 0=0 \otimes a=0, \\
& \text { 5. } a \otimes\left(\vee_{i \in I} b_{i}\right)=\vee_{i \in I}\left(a \otimes b_{i}\right), \\
& \text { 6. } a \rightarrow \wedge_{i \in I} b_{i}=\wedge_{i \in I}\left(a \rightarrow b_{i}\right), \\
& \text { 7. } 1 \rightarrow a=a, 0 \rightarrow a=1, \\
& \text { 8. } a \rightarrow(b \rightarrow c)=b \rightarrow(a \rightarrow c) . \\
& \text { 9. If } a \leq b, \text { then } \neg b \leq \neg a .
\end{aligned}
$$

Throughout this paper, a fuzzy set is identified with its membership function and takes values from a fixed complete residuated lattice $L=(L, \wedge, \vee, \otimes, \rightarrow, 0,1)$. For a nonempty set $X, L^{X}$ denotes the collection of all fuzzy subsets of $X$. Also, for all $a \in L ; \mathbf{a}(x)=a$ denotes a constant fuzzy set. Furthermore, for all $A \in$ $L^{X}$, the $\operatorname{core}(A)$ is a set of all elements $x \in X$ such that $A(x)=1$.

Definition 2.3 Let $X$ be a nonempty set. The following are induced operations of intersection $\wedge$, union $\vee$ , multiplication $\otimes$, implication $\rightarrow$ and negation $\neg$ on $L^{X}$ :

$(A \wedge B)(x)=A(x) \wedge B(x),(A \vee B)(x)=A(x) \vee B(x)$, $(A \otimes B)(x)=A(x) \otimes B(x),(A \rightarrow B)(x)=A(x) \rightarrow$ $B(x),(\neg A)(x)=\neg A(x)$.
Under the assumption about completeness of $L$, we may consider an intersection and a union of an arbitrary family of fuzzy sets.

Now, we recall the following concept of a fuzzy relation.

Definition 2.4 Let $X$ be a nonempty set. A fuzzy relation $R$ on $X$ is a fuzzy subset of $X \times X$. A fuzzy relation $R$ is called

(i) reflexive if $R(x, x)=1, \forall x \in X$;

(ii) symmetric if $R(x, y)=R(y, x), \forall x, y \in X$; and

(iii) transitive if $R(x, y) \otimes R(y, z) \leq R(x, z), \forall x, y, z \in$ $X$.

$A$ reflexive, symmetric and transitive fuzzy relation on $X$ is called a fuzzy equivalence relation.

Definition 2.5 Let $R$ be a fuzzy equivalence relation on $X$. For each $x \in X$, a fuzzy subset $E_{x}^{R}$ of $X$ such that $E_{x}^{R}(y)=R(x, y)$, for every $y \in X$, is called a fuzzy equivalence class of $R$ determined by the element $x$.

Definition 2.6 Let $\phi: X \rightarrow Y$ be a map. Then the Zadeh's fuzzy backward operator $\phi^{\leftarrow}: L^{Y} \rightarrow L^{X}$ is defined as,

$$
\phi^{\leftarrow}(B)(x)=B(\phi(x)), \forall B \in L^{Y}, \forall x \in X .
$$

\section{$3 \quad$ Fuzzy approximation spaces}

In this section, we study the concept of fuzzy approximation spaces. Further, it is shown that the lower fuzzy approximation operator preserves union under certain condition. We begin with the following.

Definition 3.1 A pair $(X, R)$ is called a fuzzy approximation space, where $X$ is a nonempty set and $R$ is a fuzzy binary relation on $X$.

If the fuzzy relation $R$ is reflexive, $(X, R)$ is called a fuzzy reflexive approximation space.

Definition 3.2 Let $(X, R)$ be a fuzzy approximation space. The pair $(\underline{R}(A), \bar{R}(A))$ of lower and upper approximation of a fuzzy set of $A \in L^{X}$ is a fuzzy rough set in $(X, R)$ where

$$
\begin{aligned}
& \underline{R}(A)(x)=\wedge_{y \in X}(R(x, y) \rightarrow A(y)), \\
& \bar{R}(A)(x)=\vee_{y \in X}(R(x, y) \otimes A(y)) .
\end{aligned}
$$

The two operators $\underline{R}, \bar{R}: L^{X} \rightarrow L^{X}$ are called the lower fuzzy approximation operator and upper fuzzy approximation operator, respectively.

Proposition 3.1 Let $(X, R)$ be a fuzzy approximation space, $a \in L, A, B \in L^{X}$ and $A_{i} \in L^{X}, i \in I$. Then 
(i) if $A \leq B$ then $\underline{R}(A) \leq \underline{R}(B)$ and $\bar{R}(A) \leq \bar{R}(B)$,

(ii) $\bar{R}\left(\vee_{i \in I} A_{i}\right)=\vee_{i \in I} \bar{R}\left(A_{i}\right), \quad \underline{R}\left(\wedge_{i \in I} A_{i}\right)=$ $\wedge_{i \in I} \underline{\underline{R}}\left(A_{i}\right)$,

(iii) $\bar{R}(\boldsymbol{a} \otimes A)=\boldsymbol{a} \otimes \bar{R}(A), \underline{R}(\boldsymbol{a} \rightarrow A)=\boldsymbol{a} \rightarrow \underline{R}(A)$,

(iv) $\bar{R}(\boldsymbol{a})=\boldsymbol{a}, \underline{R}(\boldsymbol{a})=\boldsymbol{a}$,

(v) if $L$ satisfies law of double negation, then $\neg \bar{R}(A)=$ $\underline{R}(\neg A)$.

In a fuzzy approximation space $(X, R)$, for $A, B \in L^{X}$, $\underline{R}(A \vee B) \neq \underline{R}(A) \vee \underline{R}(B)$. For example, let $L=$ $\{0, n, a, b, c, d, e, f, m, 1\}$ with $0<n<a<c<e<$ $m<1,0<n<b<d<f<m<1$ and the elements $\{a, b\},\{c, d\},\{e, f\}$ are pairwise incomparable. Then $L$ becomes a residuated lattice to the operations shown in Table 1 and Table 2.

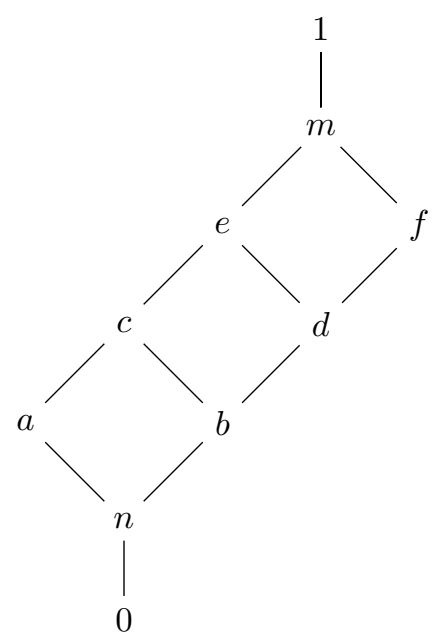

Fig: Hasse diagram of lattice $L$

\begin{tabular}{l|cccccccccc}
$\rightarrow$ & 0 & $\mathrm{n}$ & $\mathrm{a}$ & $\mathrm{b}$ & $\mathrm{c}$ & $\mathrm{d}$ & $\mathrm{e}$ & $\mathrm{f}$ & $\mathrm{m}$ & 1 \\
\hline 0 & $\mathrm{l}$ & 1 & 1 & 1 & 1 & 1 & 1 & 1 & 1 & 1 \\
$\mathrm{n}$ & $\mathrm{m}$ & 1 & 1 & 1 & 1 & 1 & 1 & 1 & 1 & 1 \\
$\mathrm{a}$ & $\mathrm{f}$ & $\mathrm{f}$ & 1 & $\mathrm{f}$ & 1 & $\mathrm{f}$ & 1 & $\mathrm{f}$ & 1 & 1 \\
$\mathrm{~b}$ & $\mathrm{e}$ & $\mathrm{e}$ & $\mathrm{e}$ & 1 & 1 & 1 & 1 & 1 & 1 & 1 \\
$\mathrm{c}$ & $\mathrm{d}$ & $\mathrm{d}$ & $\mathrm{e}$ & $\mathrm{f}$ & 1 & $\mathrm{f}$ & 1 & $\mathrm{f}$ & 1 & 1 \\
$\mathrm{~d}$ & $\mathrm{c}$ & $\mathrm{c}$ & $\mathrm{c}$ & $\mathrm{e}$ & $\mathrm{e}$ & 1 & 1 & 1 & 1 & 1 \\
$\mathrm{e}$ & $\mathrm{b}$ & $\mathrm{b}$ & $\mathrm{c}$ & $\mathrm{d}$ & $\mathrm{e}$ & $\mathrm{f}$ & 1 & $\mathrm{f}$ & 1 & 1 \\
$\mathrm{f}$ & $\mathrm{a}$ & $\mathrm{a}$ & $\mathrm{a}$ & $\mathrm{c}$ & $\mathrm{c}$ & $\mathrm{e}$ & $\mathrm{e}$ & 1 & 1 & 1 \\
$\mathrm{~m}$ & $\mathrm{n}$ & $\mathrm{n}$ & $\mathrm{a}$ & $\mathrm{b}$ & $\mathrm{c}$ & $\mathrm{d}$ & $\mathrm{e}$ & $\mathrm{f}$ & 1 & 1 \\
1 & 0 & $\mathrm{n}$ & $\mathrm{a}$ & $\mathrm{b}$ & $\mathrm{c}$ & $\mathrm{d}$ & $\mathrm{e}$ & $\mathrm{f}$ & $\mathrm{m}$ & 1
\end{tabular}

Table 1: $\rightarrow$ operation for lattice $L$.

Let $X=\{x, y, z\}$ and $A, B$ be two fuzzy sets such that $A=\{(x, b),(y, c),(z, d)\}, B=\{(x, b),(y, d),(z, d)\}$. Then $A \vee B=\{(x, b),(y, e),(z, d)\}$, since $c \vee d=e$. Now, let $R$ be fuzzy relation on $X$, as given in Table 3. Then

\begin{tabular}{l|cccccccccc}
$\otimes$ & 0 & $\mathrm{n}$ & $\mathrm{a}$ & $\mathrm{b}$ & $\mathrm{c}$ & $\mathrm{d}$ & $\mathrm{e}$ & $\mathrm{f}$ & $\mathrm{m}$ & $\mathrm{1}$ \\
\hline 0 & 0 & 0 & 0 & 0 & 0 & 0 & 0 & 0 & 0 & 0 \\
$\mathrm{n}$ & 0 & 0 & 0 & 0 & 0 & 0 & 0 & 0 & 0 & $\mathrm{n}$ \\
$\mathrm{a}$ & 0 & 0 & $\mathrm{a}$ & 0 & $\mathrm{a}$ & 0 & $\mathrm{a}$ & 0 & $\mathrm{a}$ & $\mathrm{a}$ \\
$\mathrm{b}$ & 0 & 0 & 0 & 0 & 0 & 0 & 0 & $\mathrm{~b}$ & $\mathrm{~b}$ & $\mathrm{~b}$ \\
$\mathrm{c}$ & 0 & 0 & $\mathrm{a}$ & 0 & $\mathrm{a}$ & 0 & $\mathrm{a}$ & $\mathrm{b}$ & $\mathrm{c}$ & $\mathrm{c}$ \\
$\mathrm{d}$ & 0 & 0 & 0 & 0 & 0 & $\mathrm{~b}$ & $\mathrm{~b}$ & $\mathrm{~d}$ & $\mathrm{~d}$ & $\mathrm{~d}$ \\
$\mathrm{e}$ & 0 & 0 & $\mathrm{a}$ & 0 & $\mathrm{a}$ & $\mathrm{b}$ & $\mathrm{c}$ & $\mathrm{d}$ & $\mathrm{e}$ & $\mathrm{e}$ \\
$\mathrm{f}$ & 0 & 0 & 0 & $\mathrm{~b}$ & $\mathrm{~b}$ & $\mathrm{~d}$ & $\mathrm{~d}$ & $\mathrm{f}$ & $\mathrm{f}$ & $\mathrm{f}$ \\
$\mathrm{m}$ & 0 & 0 & $\mathrm{a}$ & $\mathrm{b}$ & $\mathrm{c}$ & $\mathrm{d}$ & $\mathrm{e}$ & $\mathrm{f}$ & $\mathrm{m}$ & $\mathrm{m}$ \\
$\mathrm{l}$ & 0 & $\mathrm{n}$ & $\mathrm{a}$ & $\mathrm{b}$ & $\mathrm{c}$ & $\mathrm{d}$ & $\mathrm{e}$ & $\mathrm{f}$ & $\mathrm{m}$ & 1
\end{tabular}

Table 2: $\otimes$ operation for lattice $L$.

\begin{tabular}{c|ccc}
$\mathrm{R}$ & $\mathrm{x}$ & $\mathrm{y}$ & $\mathrm{z}$ \\
\hline $\mathrm{x}$ & $\mathrm{b}$ & $\mathrm{e}$ & $\mathrm{d}$ \\
$\mathrm{y}$ & 0 & $\mathrm{~b}$ & $\mathrm{~m}$ \\
$\mathrm{z}$ & $\mathrm{e}$ & $\mathrm{f}$ & 1
\end{tabular}

Table 3: Fuzzy binary relation on $X$.

$\underline{\mathrm{R}}(A \vee B)(x)=\wedge_{y \in X}\{R(x, y) \rightarrow(A \vee B)(y)\}$

$$
=\wedge\left\{\begin{array}{l}
b \rightarrow b \\
e \rightarrow e \\
d \rightarrow d
\end{array}\right.
$$

$$
=1 \text {. }
$$

Also,

$$
\begin{aligned}
\underline{\mathrm{R}}(A)(x)= & \wedge_{y \in X}\{R(x, y) \rightarrow(A)(y)\} \\
= & \wedge\left\{\begin{array}{l}
b \rightarrow b \\
e \rightarrow c \\
d \rightarrow d
\end{array}\right. \\
& =\wedge\left\{\begin{array}{l}
1 \\
e \\
1
\end{array}\right. \\
& =e,
\end{aligned}
$$

and

$$
\begin{aligned}
\underline{\mathrm{R}}(B)(x)= & \wedge_{y \in X}\{R(x, y) \rightarrow(B)(y)\} \\
= & \wedge\left\{\begin{array}{l}
b \rightarrow b \\
e \rightarrow d \\
d \rightarrow d
\end{array}\right. \\
& =\wedge\left\{\begin{array}{l}
1 \\
f \\
1
\end{array}\right. \\
& =f .
\end{aligned}
$$

But, $e \vee f=m \neq 1$.

Hence $\underline{\mathrm{R}}(A \vee B) \neq \underline{\mathrm{R}}(A) \vee \underline{\mathrm{R}}(B)$. 
Proposition 3.2 Let $(X, R)$ be a fuzzy approximation space and $A, B \in L^{X}$. Then $\underline{R}(A \vee B)=\underline{R}(A) \vee \underline{R}(B)$, if $\left|E_{x}^{R}\right|=1$, for every $x \in X$.

Proof: If $\left|E_{x}^{R}\right|=1$ for every $x \in X$. Then

$$
\left|E_{x}^{R}\right|=R(x, y)= \begin{cases}1, & \text { if } x=y, \\ 0, & \text { if } x \neq y .\end{cases}
$$

For every $x \in X$,

$$
\begin{gathered}
\underline{\mathrm{R}}(A \vee B)(x)=\wedge_{y \in X}\{R(x, y) \rightarrow(A \vee B)(y)\} \\
=\wedge_{y \in X} \begin{cases}(1 \rightarrow(A \vee B)(y)) & \text { if } x=y \\
(0 \rightarrow(A \vee B)(y)) & \text { if } x \neq y\end{cases} \\
=\wedge_{y \in X}\left\{\begin{array}{cc}
(A \vee B)(y) & \text { if } x=y \\
1 & \text { if } x \neq y
\end{array}\right. \\
=(A \vee B)(x) \\
=A(x) \vee B(x) .
\end{gathered}
$$

Again, for every $x \in X$,

$$
\begin{gathered}
\underline{\mathrm{R}}(A)(x)=\wedge_{y \in X}\{R(x, y) \rightarrow(A)(y)\} \\
=\wedge_{y \in X}\left\{\begin{array}{cc}
(1 \rightarrow(A)(y)) & \text { if } x=y \\
(0 \rightarrow(A)(y)) & \text { if } x \neq y
\end{array}\right. \\
=\wedge_{y \in X}\left\{\begin{array}{cc}
(A)(y) & \text { if } x=y \\
1 & \text { if } x \neq y
\end{array}\right. \\
=A(x) .
\end{gathered}
$$

Similarly, we can show that $\underline{\mathrm{R}}(B)(x)=B(x)$. Thus from above $\underline{\mathrm{R}}(A \vee B)(x)=A(x) \vee B(x)=\underline{\mathrm{R}}(A)(x) \vee$ $\underline{\mathrm{R}}(B)(x)$. Hence $\underline{\mathrm{R}}(A \vee B)=\underline{\mathrm{R}}(A) \vee \underline{\mathrm{R}}(B)$.

\section{Fuzzy natural transformations}

In this section, we introduce the concept of lower and upper fuzzy backward natural transformations. Further, we show that there is a close connection between such transformations and maps between two fuzzy approximation spaces. We begin with the following concept of an upper fuzzy backward natural transformation.

Definition 4.1 Let $(X, R)$ and $(Y, S)$ be two fuzzy approximation spaces. A one-to-one map $\phi: X \rightarrow Y$ is called an upper fuzzy backward natural transformation from $(X, R)$ into $(Y, S)$, if $\bar{R}\left(\phi^{\leftarrow}(B)\right) \leq \phi^{\leftarrow}(\bar{S}(B))$ $\forall B \in L^{Y}$.
Before stating next, we recall the following from [27].

Let $(X, R)$ and $(Y, S)$ be two fuzzy approximation spaces. A map $\phi: X \rightarrow Y$ is relation preserving if $R(x, y) \leq S(\phi(x), \phi(y)), \forall x, y \in X$.

Now, we have the following.

Proposition 4.1 Let $(X, R)$ and $(Y, S)$ be two fuzzy approximation spaces and $\phi: X \rightarrow Y$ be a one-toone map. Then $\phi$ is an upper fuzzy backward natural transformation if and only if $\phi$ is relation preserving map.

Proof: Let $\phi$ be a relation preserving map. Then for $x, y \in X, R(x, y) \leq S(\phi(x), \phi(y))$. Taking $\phi(y)=y^{\prime}$, we have $R(x, y) \leq S\left(\phi(x), y^{\prime}\right)$. Now, for $B \in L^{Y}$,

$$
\begin{aligned}
R(x, y) \otimes B(\phi(y)) \leq & S\left(\phi(x), y^{\prime}\right) \otimes \\
& B(\phi(y)) \\
\Rightarrow R(x, y) \otimes B(\phi(y)) \leq & S\left(\phi(x), y^{\prime}\right) \otimes \\
& B\left(y^{\prime}\right) \\
\Rightarrow \vee_{y \in X}\{R(x, y) \otimes B(\phi(y))\} \leq & \vee_{y \in X}\{S(\phi(x), \\
& \left.\left.y^{\prime}\right) \otimes B\left(y^{\prime}\right)\right\} \\
\Rightarrow \vee_{y \in X}\left\{R(x, y) \otimes \phi^{\leftarrow}(B)(y)\right\} \leq & \vee_{\phi(y) \in Y}\{S(\phi(x), \\
& \left.\left.y^{\prime}\right) \otimes B\left(y^{\prime}\right)\right\} \\
\Rightarrow \bar{R}\left(\phi^{\leftarrow}(B)\right)(x) \leq & \vee_{y^{\prime} \in Y}\{S(\phi(x), \\
& \left.\left.y^{\prime}\right) \otimes B\left(y^{\prime}\right)\right\} \\
\Rightarrow \bar{R}\left(\phi^{\leftarrow}(B)\right)(x) \leq & \bar{S}(B)(\phi(x)) \\
\Rightarrow \bar{R}\left(\phi^{\leftarrow}(B)\right)(x) \leq & \phi^{\leftarrow}(\bar{S}(B))(x)
\end{aligned}
$$

Thus $\bar{R}\left(\phi^{\leftarrow}(B)\right) \leq \phi^{\leftarrow}(\bar{S}(B))$. Hence $\phi$ is an upper fuzzy backward natural transformation.

Conversely, let $\phi$ be an upper fuzzy backward natural transformation. Now, for $y \in X$ and $y^{\prime} \in Y$,

$$
1_{y^{\prime}}(\phi(y))= \begin{cases}1, & \text { if } y^{\prime}=\phi(y), \\ 0, & \text { if } y^{\prime} \neq \phi(y) .\end{cases}
$$

Also, for $x \in X$,

$$
\begin{aligned}
\bar{R}\left(\phi^{\leftarrow}\left(1_{y^{\prime}}\right)\right)(x) & =\vee_{y \in X}\left\{R(x, y) \otimes\left(1_{y^{\prime}}\right)(\phi(y)\}\right. \\
& =R(x, y), \text { when } \phi(y)=y^{\prime} .
\end{aligned}
$$

Thus $\bar{R}\left(\phi^{\leftarrow}\left(1_{y^{\prime}}\right)\right)(x)=R\left(x, \phi^{-1}\left(y^{\prime}\right)\right)$.

Again, for $x \in X$,

$$
\begin{aligned}
\phi^{\leftarrow}\left(\bar{S}\left(1_{y^{\prime}}\right)\right)(x) & =\bar{S}\left(1_{y^{\prime}}\right)(\phi(x)) \\
& =\vee_{z^{\prime} \in Y}\left\{S\left(\phi(x), z^{\prime}\right) \otimes\left(1_{y^{\prime}}\right)\left(z^{\prime}\right)\right\} \\
& =S\left(\phi(x), y^{\prime}\right) \text { when } y^{\prime}=z^{\prime} .
\end{aligned}
$$

Since $\phi$ is a one-to-one map, $\phi(y)=y^{\prime}=z^{\prime}$ and $\phi^{-1}\left(y^{\prime}\right)=y$. Also $\phi$ being an upper fuzzy 
backward natural transformation, $\bar{R}\left(\phi^{\leftarrow}\left(1_{y^{\prime}}\right)\right)(x) \leq$ $\phi^{\leftarrow}\left(\bar{S}\left(1_{y^{\prime}}\right)\right)(x)$, or $R\left(x, \phi^{-1}\left(y^{\prime}\right)\right) \leq S\left(\phi(x), y^{\prime}\right)$, or that $R(x, y) \leq S(\phi(x), \phi(y))$. Hence $\phi$ is relation preserving map.

Now, we introduce the following concept of lower fuzzy backward natural transformation.

Definition 4.2 Let $(X, R)$ and $(Y, S)$ be two fuzzy approximation spaces. A one-to-one map $\phi: X \rightarrow Y$ is called a lower fuzzy backward natural transformation from $(X, R)$ into $(Y, S)$, if $\phi^{\leftarrow}(\underline{S}(B)) \leq \underline{R}\left(\phi^{\leftarrow}(B)\right)$ $\forall B \in L^{Y}$

Following is required to establish the relationship similar to Proposition 4.1 for lower fuzzy backward natural transformation.

Lemma 4.1 Let $(X, R)$ and $(Y, S)$ be two fuzzy approximation spaces and $\phi: X \rightarrow Y$ be an upper fuzzy backward natural transformation if and only if $\phi$ is a lower fuzzy backward natural transformation, provided $L$ satisfies the double negation law.

Proof: For all $x \in X$ and $B \in L^{Y}$

$$
\begin{aligned}
\underline{R}\left(\neg\left(\phi^{\leftarrow}(\neg B)\right)\right)(x)= & \wedge_{y \in X}\{R(x, y) \rightarrow \\
& \neg\left(\phi^{\leftarrow}(\neg(B))(y)\right\} \\
= & \wedge_{y \in X}\{R(x, y) \rightarrow \\
& \neg(\neg(B))(\phi(y))\} \\
= & \wedge_{y \in X}\{R(x, y) \rightarrow B(\phi(y))\} \\
= & \wedge_{y \in X}\left\{R(x, y) \rightarrow\left(\phi^{\leftarrow}(B)(y)\right\}\right. \\
= & \underline{R}\left(\phi^{\leftarrow}(B)\right)(x) .
\end{aligned}
$$

Since $\phi$ is an upper natural backward fuzzy transformation,

$$
\begin{aligned}
\phi^{\leftarrow}(\bar{S}(B))(x) & \geq \bar{R}\left(\phi^{\leftarrow}(B)\right)(x) \\
\Longleftrightarrow \bar{S}(B))(\phi(x)) & \geq \bar{R}\left(\phi^{\leftarrow}(B)\right)(x) \\
\Longleftrightarrow \neg \bar{S}(B)(\phi(x)) & \leq \neg \bar{R}\left(\phi^{\leftarrow}(B)\right)(x) \\
\Longleftrightarrow(\neg \bar{S}(B))(\phi(x)) & \leq \neg \bar{R}\left(\phi^{\leftarrow}(B)\right)(x) \\
\Longleftrightarrow \phi^{\leftarrow}(\neg \bar{S}(B))(x) & \leq \neg \bar{R}\left(\phi^{\leftarrow}(B)\right)(x) \\
\Longleftrightarrow \phi^{\leftarrow}(\underline{S}(\neg B))(x) & \leq \underline{R}\left(\neg\left(\phi^{\leftarrow}(B)\right)\right)(x)
\end{aligned}
$$

Replacing $B$ by $\neg B$ and using $\neg \neg B=B$, we have $\phi^{\leftarrow}(\underline{S}(B))(x) \leq \underline{R}\left(\neg\left(\phi^{\leftarrow}(\neg B)\right)\right)(x)$, or that $\phi^{\leftarrow}(\underline{S}(B))(x) \leq \underline{R}\left(\phi^{\leftarrow}(B)\right)(x)$. Thus $\phi^{\leftarrow}(\underline{S}(B)) \leq \underline{R}\left(\phi^{\leftarrow}(B)\right)$. Hence $\phi$ is a lower natural backward fuzzy transformation.

Finally, we have the following.

Proposition 4.2 Let $(X, R)$ and $(Y, S)$ be two fuzzy approximation spaces and $\phi: X \rightarrow Y$ be a one-toone map. Then $\phi$ is a lower fuzzy backward natural transformation if and only if $\phi$ is relation preserving, provided $L$ satisfies the double negation law.
Proof: Follows from the Proposition 4.1 and Lemma 4.1 .

\section{$5 \quad$ Fuzzy transformation systems vs. fuzzy approximation spaces}

In this section, we introduce the concepts of upper/lower fuzzy transformation system which are slight modification of similar concepts introduced in [9]. Interestingly, we show that there is a bijective correspondence between upper/lower fuzzy transformation systems and fuzzy reflexive approximation spaces. We begin with the following.

Definition 5.1 Let $X$ be a nonempty set and $G$ : $L^{X} \rightarrow L^{X}$ be a map. Then the system $(X, G)$ is called upper fuzzy transformation system if

(i) for each $A \in L^{X}, x \in X, A(x) \leq G(A)(x)$,

(ii) for each $\left\{A_{i}: i \in I\right\} \in L^{X}, G\left(\vee_{i \in I} A_{i}\right)=$ $\vee_{i \in I} G\left(A_{i}\right)$

(iii) for each $a \in L, A \in L^{X}, G(\boldsymbol{a} \otimes A)=\boldsymbol{a} \otimes G(A)$, (iv) core $\left(G\left(1_{x}\right)\right) \neq \emptyset$, where $1_{x}$ is the characteristic function of $\{x\}$ in $X$ and $\emptyset$ is an empty set.

Now we recall following from [9]

Lemma 5.1 Let $A \in L^{X}$. Then $A=\vee_{x \in X} \boldsymbol{A}(\boldsymbol{x}) \otimes 1_{x}$, where $\boldsymbol{A}(\boldsymbol{x})$ is a constant function with constant value $A(x)$.

Theorem 5.1 Given an upper fuzzy transformation system $(X, G)$, there exists a fuzzy reflexive approximation space $(X, R)$ such that $G=\bar{R}$. Conversely, given a fuzzy reflexive approximation space $(X, R)$, $(X, \bar{R})$ is an upper fuzzy transformation system.

Proof: $(1) \Rightarrow(2)$. Let $(X, G)$ be upper fuzzy transformation system. For $x, y \in X$, let $R(y, x)=G\left(1_{x}\right)(y)$. Then

$$
\begin{gathered}
\bar{R}\left(1_{x}\right)(y)=\vee_{z \in X} R(y, z) \otimes 1_{x}(z) \\
=\vee_{z \in X}\left\{\begin{array}{rr}
R(y, z) \otimes 1 & \text { if } z=x \\
R(y, z) \otimes 0 & \text { if } z \neq x
\end{array}\right. \\
=R(y, x)=G\left(1_{x}\right)(y) .
\end{gathered}
$$

Thus $\bar{R}\left(1_{x}\right)=G\left(1_{x}\right)$.

Now, for $x \in X, R(x, x)=G\left(1_{x}\right)(x) \geq 1_{x}(x)=1$, or $R(x, x)=1$. Thus $R$ is a fuzzy reflexive relation. Again, for $y \in X$ and $A \in L^{X}$,

$$
\begin{aligned}
\bar{R}(A)(y) & =\bar{R}\left\{\vee_{x \in X} \mathbf{A}(\mathbf{x}) \otimes 1_{x}\right\}(y) \\
& =\vee_{z \in X}\left\{R(y, z) \otimes\left\{\vee_{x \in X} \mathbf{A}(\mathbf{x}) \otimes 1_{x}\right\}(z)\right\} \\
& =\vee_{z \in X} \vee_{x \in X}\left\{R(y, z) \otimes\left(A(x) \otimes 1_{x}(z)\right)\right\} \\
& =\vee_{x \in X}\left\{A(x) \otimes \vee_{z \in X}\left\{R(y, z) \otimes 1_{x}(z)\right\}\right\}
\end{aligned}
$$




$$
\begin{aligned}
& =\vee_{x \in X}\left\{A(x) \otimes G\left(1_{x}\right)(y)\right\} \\
& =G\left(\vee_{x \in X} \mathbf{A}(\mathbf{x}) \otimes 1_{x}\right)(y) \\
& =G(A)(y) .
\end{aligned}
$$

Hence $\bar{R}(A)=G(A)$ for all $A \in L^{X}$.

$(2) \Rightarrow(1)$. Let $(X, R)$ be a fuzzy reflexive approximation space, and $\bar{R}: L^{X} \rightarrow L^{X}$ be an upper fuzzy approximation operator. Then

(i) for each, $A \in L^{X}, x \in X,, A(x) \leq \bar{R}(A)(x)$,

(ii) for each $\left\{A_{i}: i \in I\right\} \in L^{X}, \bar{R}\left(\vee_{i \in I} A_{i}\right)=$ $\vee_{i \in I} \bar{R}\left(A_{i}\right)$,

(iii) for each $a \in L$ and $A \in L^{X}, \bar{R}(\mathbf{a} \otimes A)=\mathbf{a} \otimes \bar{R}(A)$,

(iv) for all $y \in X$,

$$
\begin{aligned}
\bar{R}\left\{1_{x}\right\}(y) & =\vee_{z \in X}\left\{R(y, z) \otimes 1_{x}(z)\right\} \\
& =R(y, x) \otimes 1_{x}(x) \\
& =R(y, x) \otimes 1 \\
& =R(y, x) .
\end{aligned}
$$

Since $R$ is reflexive, core $\bar{R}\left\{1_{x}\right\} \neq \emptyset$.

Hence $(X, \bar{R})$ is an upper fuzzy transformation system.

Similar to the concept of upper fuzzy transformation system, now we introduce the following concept of a lower fuzzy transformation system.

Definition 5.2 Let $X$ be a nonempty set and $H$ : $L^{X} \rightarrow L^{X}$ be a map. Then the system $(X, H)$ is called lower fuzzy transformation system if

(i) for each $A \in L^{X}, x \in X, H(A)(x) \leq A(x)$,

(ii) for each $\left\{A_{i}: i \in I\right\} \in L^{X}, H\left(\wedge_{i \in I} A_{i}\right)=$ $\wedge_{i \in I} H\left(A_{i}\right)$,

(iii) for each $a \in L, A \in L^{X}, H(\boldsymbol{a} \rightarrow A)=\boldsymbol{a} \rightarrow H(A)$, (iv) $\operatorname{core}\left(\neg H\left(\neg 1_{x}\right)\right) \neq \emptyset$.

Before stating next, we recall the following from [9].

Lemma 5.2 Let $A \in L^{X}$. Then $\neg A=\wedge_{x \in X} \boldsymbol{A}(\boldsymbol{x}) \rightarrow$ $\left(1_{x} \rightarrow 0\right)$, where $\boldsymbol{A}(\boldsymbol{x})$ is a constant function with constant value $A(x)$.

Finally, we have the following.

Theorem 5.2 Let $L$ be a complete residuated lattice which satisfies the double negation law. Then for a given lower fuzzy transformation system $(X, H)$, there exists a fuzzy reflexive approximation space $(X, R)$ such that $H=\underline{R}$. Conversely, given a fuzzy reflexive approximation space $(X, R),(X, \underline{R})$ is a lower fuzzy transformation system.

Proof: $(1) \Rightarrow(2)$ Let $(X, H)$ be lower fuzzy transformation system. For $x, y \in X$, let $R(y, x)=$ $\neg H\left(\neg 1_{x}\right)(y)$. Then

$$
\underline{R}\left(\neg 1_{x}\right)(y)=\wedge_{z \in X} R(y, z) \rightarrow\left(\neg 1_{x}\right)(z)
$$

$$
\begin{aligned}
& =\wedge_{z \in X} \begin{cases}R(y, z) \rightarrow 0 & \text { if } z=x \\
R(y, z) \rightarrow 1 & \text { if } z \neq x\end{cases} \\
& =\neg R(y, x)=H\left(\neg 1_{x}\right)(y) .
\end{aligned}
$$

Thus $\underline{R}\left(\neg 1_{x}\right)=H\left(\neg 1_{x}\right)$.

Now, for $x \in X, R(x, x)=\neg H\left(\neg 1_{x}\right)(x) \geq$ $\neg\left(\neg 1_{x}\right)(x)=1_{x}(x)=1$, or $R(x, x)=1$. Thus $R$ is a fuzzy reflexive relation. Again, for $y \in X$ and $A \in L^{X}$,

$$
\begin{aligned}
\underline{R}(A)(y)= & \underline{R}\left\{\wedge_{x \in X} \neg \mathbf{A}(\mathbf{x}) \rightarrow\left(\neg 1_{x}\right)\right\}(y) \\
= & \wedge_{x \in X}\left\{\underline{R}\left\{\neg \mathbf{A}(\mathbf{x}) \rightarrow\left(\neg\left(1_{x}\right)\right\}\right\}(y)\right. \\
= & \wedge_{x \in X} \wedge_{z \in X}\{R(y, z) \rightarrow \\
& \left.\left\{\neg \mathbf{A}(\mathbf{x}) \rightarrow\left(\neg 1_{x}\right)\right\}(z)\right\} \\
= & \wedge_{x \in X} \wedge_{z \in X}\{\neg A(x) \rightarrow \\
& \left.\left\{R(y, z) \rightarrow \neg\left(1_{x}\right)(z)\right\}\right\} \\
= & \wedge_{x \in X}\{\neg A(x) \rightarrow \\
& \left.\wedge_{z \in X}\left\{R(y, z) \rightarrow \neg\left(1_{x}\right)(z)\right\}\right\} \\
= & \wedge_{x \in X}\left\{\neg A(x) \rightarrow \underline{R}\left(\neg 1_{x}\right)(y)\right\} \\
= & \wedge_{x \in X}\left\{\neg A(x) \rightarrow H\left(\neg 1_{x}\right)(y)\right\} \\
= & \wedge_{x \in X} H\left\{\neg \mathbf{A}(\mathbf{x}) \rightarrow\left(\neg 1_{x}\right)\right\}(y) \\
= & H\left\{\wedge_{x \in X} \neg \mathbf{A}(\mathbf{x}) \rightarrow\left(\neg 1_{x}\right)\right\}(y) \\
= & H(A)(y) .
\end{aligned}
$$

Hence $\underline{R}(A)=H(A)$ for all $A \in L^{X}$.

$(2) \Rightarrow(1)$. Let $(X, R)$ be a fuzzy reflexive approximation space, and $\underline{R}: L^{X} \rightarrow L^{X}$ be lower fuzzy approximation operator. Then

(i) for each $A \in L^{X}, \underline{R}(A)(x) \leq A(x)$

(ii) for each $\left\{A_{i}: i \in I\right\} \in L^{X}, \underline{R}\left(\wedge_{i \in I} A_{i}\right)=$ $\wedge_{i \in I} \underline{R}\left(A_{i}\right)$,

(iii) for each $a \in L$ and $A \in L^{X}, \underline{R}(\mathbf{a} \rightarrow A)=\mathbf{a} \rightarrow$ $\underline{R}(A)$,

(iv) for $y \in X$,

$$
\begin{aligned}
& \neg \underline{R}\left(\neg 1_{x}\right)(y)=\left.\neg\left\{\wedge_{a \in X} R(y, a) \rightarrow\left(\neg 1_{x}\right)(a)\right)\right\} \\
&=\neg \wedge_{a \in X} \begin{cases}(R(y, a) \rightarrow 0) & \text { if } a=x \\
(R(y, a) \rightarrow 1) & \text { if } a \neq x\end{cases} \\
&=\neg \wedge_{a \in X}\left\{\begin{array}{cc}
\neg R(y, a) & \text { if } a=x \\
1 & \text { if } a \neq x
\end{array}\right. \\
&=\neg \neg R(y, x) \\
&=R(y, x) .
\end{aligned}
$$

Since $R$ is reflexive, $\operatorname{core}\left(\neg R\left(\neg 1_{x}\right)\right) \neq \emptyset$. Hence $(X, R)$ is a lower fuzzy transformation system.

\section{Conclusions}

In this paper, we have established an interesting relationship between fuzzy transformation systems and 
fuzzy approximation spaces induced by fuzzy reflexive relations. In view of the studied done in [15], it can be seen that the relationship established in [9] between $F$-transforms and fuzzy transformation systems is an special case of the results obtained in this paper.

\section{References}

[1] R. Bělohlávek, Fuzzy relational systems: Foundations and principles, Kluwer Academic/Plenum Publishers, New York, (2002).

[2] R. Blount, C. Tsinakis, The structure of residuated lattices, International Journal of Algebra and Computation 13 (2003) 437-461.

[3] M. Ćirić, J. Ignjatović, S. Bogdanović, Fuzzy equivalence relations and their equivalence classes, Fuzzy Sets and Systems 158 (2007) 12951313.

[4] L. D́eer, N. Verbiest, C. Cornelis, L. Godo, A comprehensive study of implicator-conjunctorbased and noise-tolerant fuzzy rough sets: definitions, properties and robustness analysis, Fuzzy sets and systems 275 (2015) 1-38.

[5] D. Dubois, H. Prade, Rough fuzzy sets and fuzzy rough sets, International Journal of General System 17 (1990) 191-209.

[6] J. A. Goguen, L-fuzzy sets, Journal of Mathematical Analysis and Applications 18 (1967) 145-174.

[7] M. Kondo, On the structures of generalized rough sets, Information Sciences 176 (2006) 586-600.

[8] J. S. Mi, Y. Leung, H. Y. Zhao, T. Feng, Generalized fuzzy rough sets determined by a triangular norm, Information Sciences 178 (2008) 3203-3213.

[9] J. Močkoř, Axiomatic of lattice valued Ftransform, Fuzzy sets and systems 342 (2018) 5366.

[10] N. N. Morsi, M. M. Yakout, Axiomatics for fuzzy rough sets, Fuzzy sets and systems 100 (1998) 327-342.

[11] Z. Pawlak, Rough sets, International Journal of Computer and Information Sciences 11 (1982) 341-356.

[12] D. Pei, Fuzzy logic algebras on residuated Lattices, Southeast Asian Bulletin of Mathematics 28 (2004) 519-531.

[13] I. Perfilieva, Fuzzy transforms: theory and applications, Fuzzy sets and systems 157 (2006) 9931023.
[14] I. Perfilieva, Fuzzy transforms: a challenge to conventional transforms, Advances in Imaging and Electron Physics 147 (2007) 137-196.

[15] I. Perfilieva, A.P. Singh, S.P. Tiwari, On the relationship among $F$-transform, fuzzy rough set and fuzzy topology Soft Computing, 21 (2017) 35133523 .

[16] K. Qin, J. Yang Z. Pei, Generalized rough sets based on reflexive and transitive relations, Information Sciences 178 (2008) 4138-4141.

[17] A. M. Radzikowska, E. E. Kerre, A comparative study of fuzzy rough sets, Fuzzy sets and systems 126 (2002) 137-155.

[18] A. M. Radzikowska, E. E. Kerre, Fuzzy rough sets based on residuated lattices, in: Transactions on Rough Sets II, in: LNCS, Springer-Verlag, 3135 (2004) 278-296.

[19] Y. H. She, G. J. Wang, An axiomatic approach of fuzzy rough sets based on residuated lattices, Computer and Mathematics with Applications 58 (2009) 189-201.

[20] L. Stefanini, F-transform with parametric generalized fuzzy partitions, Fuzzy sets and systems 180 (2011) 98-120.

[21] S.P. Tiwari, A.K. Srivastava, Fuzzy rough sets, fuzzy preorders and fuzzy topologies, Fuzzy Sets and Systems 210(2013) 63-68.

[22] C. Y. Wang, B. Q. Hu, Granular variable precision fuzzy rough sets with general fuzzy relations, Fuzzy sets and systems 275 (2015) 39-57.

[23] W. Z. Wu, Y. Leung, M. W. Shao, Generalized fuzzy rough approximation operators determined by fuzzy implicators, International Journal of Approximate Reasoning 54 (2013) 1388-1409.

[24] Y. Yao, J. Mi, Z. Li, A novel variable precision $(\theta, \sigma)$-fuzzy rough set model based on fuzzy granules, Fuzzy sets and systems 236 (2014) 58-72.

[25] Y. Y. Yao, Constructive and algebraic methods of the theory of rough sets, Information Sciences 109 (1998) 21-47.

[26] L. A. Zadeh, The Concept of a linguistic variable and its application to approximation reasoning-I, Information Sciences 8 (1975) 199-249.

[27] Y. Zhao, Homomorphisms between fuzzy approximation spaces based on residuated lattice, Journal of Applied Mathematics 2014 (2014). 